\title{
Effect of bacteria density and accumulated inert solids on the effluent pollutant concentrations predicted by a Constructed Wetlands model
}

\author{
Roger Samsóa, Jordi Blázquez ${ }^{\mathrm{a}}$, Núria Agullóa, Joan Grau ${ }^{\mathrm{b}}$, Ricardo \\ Torres $^{\mathrm{b}}$, Joan García ${ }^{\mathrm{a}}$ \\ ${ }^{a}$ GEMMA - Group of Environmental Engineering and Microbiology, Department of \\ Hydraulic, Maritime and Environmental Engineering, Universitat Politècnica de \\ Catalunya-BarcelonaTech, c/ Jordi Girona, 1-3, Building D1, E-08034, Barcelona, Spain. \\ ${ }^{b}$ Fluid Mechanics Department, Universitat Politècnica de Catalunya-BarcelonaTech, c/ \\ Urgell 187. E-08036, Barcelona, Spain.
}

\section{Abstract}

Constructed wetlands are a widely adopted technology for the treatment of wastewater in small communities. The understanding of their internal functioning has increased at an unprecedented pace over recent years, in part thanks to the use of mathematical models. BIO_PORE model is one of the most recent models developed for constructed wetlands. This model was built in the COMSOL Multiphysics ${ }^{T M}$ software and implements the biokinetic expressions of Constructed Wetlands Model 1 (CWM1) to describe the fate and transport of organic matter, nitrogen and sulphur in horizontal subsurfaceflow constructed wetlands. In previous studies, CWM1 was extended with the inclusion of two empirical parameters $\left(M_{\text {bio_max }}\right.$ and $\left.M_{c a p}\right)$ that proved to be essential to provide realistic bacteria growth rates and dynamics. The aim of the current work was to determine the effect of these two parameters on the effluent pollutant concentrations predicted by the model. To that end, nine simulations, each with a different $M_{\text {bio_max }}-M_{\text {cap }}$ pair, were launched on 
a high-end multi-processor computer and the effluent COD and ammonia nitrogen concentrations obtained on each simulation were qualitatively compared among them. Prior to this study, a finite element mesh optimization procedure was carried out to reduce computational cost. Results of the mesh optimization procedure indicated that among the 5 tested meshes of different element size, the mesh utilized for this model in previous studies represented a fair compromise between output accuracy and computation time. Results of the sensitivity analysis showed that the value of $M_{c a p}$ has a dramatic effect on the simulated effluent concentrations of COD and ammonia nitrogen, which clearly decreased for increasing values of this parameter. On the other hand, $M_{\text {bio_max }}$ was also sensitive, but its effects on the model output were less important and no clear relation could be established between its value and the simulated effluent concentration of COD and ammonia nitrogen.

Keywords: Local sensitivity, mesh optimization, bacteria, growth, parallel computing, batch

\section{Introduction}

2 Constructed Wetlands (CWs) are wastewater treatment systems usu3 ally applied for communities of less than 2000PE. This technology provides 4 comparable treatment efficiencies with significantly lower energy and main5 tenance requirements than conventional technologies (García et al., 2010; 6 Puigagut et al., 2007).

7 However, and due to the diversity and complexity of the physic-chemical 8 and biological processes occurring within CWs, their functioning is far less 9 well understood than that of activated sludge systems. To bridge this knowl- 
edge gap, several mathematical models have been developed in recent years to simulate CWs functioning (Meyer et al., 2014; Samsó et al., 2014b).

The BIO_PORE model is one of such models and was developed in COMSOL Multiphysics ${ }^{T M}$, a commercial finite elements (FE) simulation platform (Meyer et al., 2014; Samsó and García, 2014a; Samsó et al., 2014b; Samsó and García, 2013a,b). This model aims at describing the hydraulics and hydrodynamics of CWs, as well as the removal of the most common pollutants found in wastewater. To that end, it implements the biokinetic model Constructed Wetlands Model 1 (CWM1) (Langergraber et al., 2009), which describes the fate of organic mater, nitrogen and sulphur. This biokinetic model is based on the formulation of the well-known Activated Sludge Model series (ASMs) for aerobic and anoxic processes (Henze et al., 2000) and on the Anaerobic Digestion Model 1 (ADM1) to describe anaerobic processes (Batstone et al., 2002).

In BIO_PORE two logistic functions are added to the original formulation of CWM1, which involve two new empirical parameters $M_{\text {bio_max }}$ and $M_{c a p}$ (Samsó and García, 2013a). These two parameters represent, respectively, the maximum microbial biomass (carrying capacity) and the maximum amount of particulate solids that can be maintained in a representative volume of granular material. The function involving $M_{\text {bio-max }}$ has already been used in several bioclogging studies (Brovelli et al., 2009) and adds a negative feedback term to the growth of all bacteria groups to prevent their unlimited growth in areas where substrates concentrations are high. On the other hand, the expression involving parameter $M_{c a p}$ also adds a negative feedback term to the growth equations, but in this case it decreases the growth rate 
of bacteria due to the progressive accumulation of inert solids in the pore space of the granular media (Samsó and García, 2014a). Our previous studies proved the importance of these two functions in order to obtain realistic bacteria concentrations within the granular media (Samsó and García, 2014a; Samsó and García, 2013a). As bacterial communities play a major role on the treatment of wastewater in CWs, these two functions also improved the model predictions regarding effluent pollutant concentrations.

However, in these previous studies a sensitivity analysis of parameters $M_{\text {bio_max }}$ and $M_{c a p}$ was not carried out and so their effect on the model output could not be evaluated. A parameter with high sensitivity is one for which small changes in its value produce large variation in a certain output of the model. On the contrary, low sensitivity parameters are those which do not affect model outputs even for large changes on their value. In this context, the main objective of the current work was to evaluate the sensitivity of $M_{b i o \_m a x}$ and $M_{c a p}$ on the effluent pollutant concentrations of COD and ammonia and ammonium nitrogen predicted by the model. To that end, the BIO_PORE model was used with the same domain, parameter values and initial and boundary conditions than in our previous paper in which the model was calibrated (Samsó and García, 2013a). Due to the large computational cost associated with solving the model for a simulated period of an entire year of operation of a wetland (up to 16 hours for dense finite elements (FE) meshes with a current desktop computer), and due to the large number of simulations needed for the current and for further studies, a previous mesh optimization procedure was carried out. The objective of this part of the study was to find the FE mesh which would provide the best compromise 
between numerical solutions accuracy and computational cost.

The two empirical parameters discussed in this work are essential to obtain realistic bacteria concentrations when simulating CWs and this study shows how they affect the effluent pollutant concentrations predicted by the BIO_PORE model. In this work we also exploited the batch and parallel computation functionalities of COMSOL Multiphysics ${ }^{T M}$ on a high-end multi-processor computer which is easily justified by the large number of simulations performed.

\section{Methods}

The local parameter sensitivity analysis and the mesh optimization procedure were performed using the exact same domain, parameter values and boundary and initial conditions as in Samsó and García (2013a). For this reason, only the basic equations of the BIO_PORE model are described in this section. For an in-depth description of all model equations the reader is referred to the original source. All simulations performed in this study were run for the entire first year of operation of a pilot wetland.

\subsection{BIO_PORE model description}

\subsubsection{Governing equations}

In BIO_PORE model, the saturated porous media flow is described using the Darcy equation (Eq. 1).

$$
q_{i}=-K_{i j} \frac{\partial H}{\partial x_{j}}
$$

Where, $q_{i}$ is the specific discharge $\left[L T^{-1}\right], K_{i j}$ is the saturated hydraulic conductivity tensor $\left[L T^{-1}\right]$, and $\frac{\partial H}{\partial x_{j}}$ the hydraulic gradient vector (unitless). 
Since in CWs both saturated and unsaturated conditions coexist, the $D e$ formed Geometry node of COMSOL Multiphysics ${ }^{T M}$ was used to dynamically adjust the top boundary of the model domain to the simulated shape and location of the water table.

The fate and transport of the aqueous phase (mobile) wastewater components of CWM1 (Table 1) are described with reactive transport equations, one for each component, in which the reactive term accounts for the production/consumption of the substrate through microbial activity (Eq. 2)(Clement et al., 1998).

$$
\frac{\partial C_{k}}{\partial t}=\frac{\partial}{\partial x_{i}}\left(D_{i j} \frac{\partial C_{k}}{\partial x_{j}}\right)-\frac{\partial}{\partial x_{i}}\left(q_{i} C_{k}\right)+r_{r}-r_{a t t}+r_{d e t}+s_{s}
$$

Where $k=1,2 . . m$

Where, $m$ is the total number of aqueous phase species (dissolved and particulate, see Table 1), $C_{k}\left[M L^{-3}\right]$ is the concentration of the $k^{\text {th }}$ aqueous phase species, $D_{i j}\left[L^{2} T^{-1}\right]$ is the hydrodynamic dispersion tensor. $q_{i}\left[L T^{-1}\right]$ is the specific discharge and acts as the coupling variable between equations 1 and 2. $r_{r}\left[M L^{-3} T^{-1}\right]$ is the reaction rate of the $k^{\text {th }}$ species in the aqueous phase. $r_{\text {att }}\left[M L^{-3} T^{-1}\right]$ and $r_{\text {det }}\left[M L^{-3} T^{-1}\right]$ are attachment and detachment rates, respectively, and are used to simulate mass exchanges between the aqueous and the solid phases of particulate components $X_{S}$ and $X_{I} . s_{s}$ $\left[M L^{-3} T^{-1}\right]$ is the source/sink term, which represents external sources or sinks of species $C_{k}$. This last term is only used to simulate oxygen release and nutrients uptake through plant roots (see Samsó and García (2013a) for more details).

On the other hand, Eq. (3) describes the fate of the solid phase (immobile) 
species (Table 1):

$$
\frac{d \check{C}_{l}}{d t}=\check{r}_{r}+r_{a t t}-r_{d e t}
$$

Where $l=1,2, . . n$

Where, $n$ is the total number of solid phase species (particulate only), $\check{C}_{l}\left[M L^{-3}\right]$ is the concentration of the $l^{\text {th }}$ species and $\check{r}_{r}\left[M L^{-3} T^{-1}\right]$ is the reaction rate of the $l^{\text {th }}$ species on the solid phase.

The growth and decay rates of each bacteria group included in CWM1 (Table 2) are described using Monod expressions (Monod, 1949), to which the product of two logistic expressions was added (Eq. 4):

$$
f_{G L}=\left(1-\frac{M_{\text {bio }}}{M_{\text {bio_max }}}\right)\left(1-\frac{M_{X_{I f}}}{M_{\text {cap }}}\right)
$$

Where, $M_{\text {bio_max }}$ and $M_{c a p}[M]$ are two empirical parameters representing, respectively, the maximum microbial biomass (carrying capacity) and the maximum amount of particulate solids that can be maintained in a representative volume of granular material. On the other hand, $M_{b i o}$ and $M_{X_{I f}}$ $[M]$ are, respectively, the sum of the total microbial biomass and the actual mass of immobile $X_{I}$ present in the representative volume.

Table 3 shows the biokinetic processes rates of the BIO_PORE model resulting from the inclusion of Eq. 4 to the original formulation of CWM1.

Notice that all kinetic parameters of CWM1 are interpolated to account for water temperature variations. 


\subsubsection{Model domain}

The model domain corresponds to a longitudinal section of wetland C2 of the pilot system described in García et al. (2004a,b) (Figure 1). This wetland was $10.3 \mathrm{~m}$ long and $5.3 \mathrm{~m}$ wide, with a bottom slope of $1 \%$. The granular media consisted of fine granitic gravel $\left(D_{60}=3.5 \mathrm{~mm}, C_{u}=1.7\right.$, initial porosity $n=40 \%$ ) with a depth of approximately $0.6 \mathrm{~m}$ at the inlet and $0.7 m$ at the outlet.

\subsubsection{Initial and boundary conditions}

Experimentally measured flow-rates, ranging from 1.1 to $2.45 \mathrm{~m}^{3} \cdot \mathrm{d}^{-1}$ were imposed at boundary 3 (inlet) and a hydraulic head of $0.5 \mathrm{~m}$ at boundary 5 (outlet). An hydraulic head of $0.5 \mathrm{~m}$ was set as the initial condition for the Darcy equation.

For the transport equations, inflow concentrations of the components listed in Table 1, which were obtained from field measurements (see Section 2.1.4), were imposed at boundary 3. An outflow boundary condition was imposed at boundary 5. The initial concentrations of all substrates within the wetland were set to $10 \mathrm{mg} \cdot \mathrm{L}^{-1}$.

The initial concentrations of the different bacteria groups within the wetland were set to $1 \mathrm{mg} \cdot L^{-1}$ to recreate start-up conditions.

\subsubsection{Experimental data and parameter values}

The experimental data measured along the first year of operation of the pilot wetland and used to feed the model consisted of: 39 values of flow rate, 32 values of water temperature, 31 values of inflow COD and 33 values of inflow $\mathrm{NH}_{4}-\mathrm{N}$. The fractioning of the inflow COD was made using 
recommended values for primary effluents in ASMs (Henze et al., 2000): 15\% $S_{F}, 50 \% X_{S m}\left(0 \% X_{S f}\right), 20 \% S_{A}, 5 \% S_{I}$ and $10 \% X_{I m}\left(0 \% X_{I f}\right) .28$ values of COD and 34 of $\mathrm{NH}_{4}-\mathrm{N}$ were measured at the outlet of the pilot wetland during the same period of time.

The inflow concentrations of the rest of components of CWM1 $\left(0 \mathrm{mg} \cdot \mathrm{L}^{-1}\right.$ for $S_{N O}$ and $S_{H 2 S}$, and $72 \mathrm{mg} \cdot L^{-1}$ for $\left.S_{S O 4}\right)$ correspond to mean values measured from different samples extracted from the same pilot wetland by García et al. (2004b). Inflow oxygen concentration was set to zero, since DO concentration in primary treated wastewater is usually very small (Tyroller et al., 2010).

Values of the hydraulic and hydrodynamic parameters obtained by Samsó and García (2013a) and utilised for all simulations are shown in Table 4.

\subsection{Mesh Optimization}

After a previous detailed study with simplified versions of the model (progressively increasing the number of functional bacterial groups)(results not shown), 5 triangular meshes of different elements densities (Table 5) were chosen to perform the mesh optimization of the complete model (with all bacteria groups listed in Table 2). Among those meshes, $M_{0.1}$ was the coarsest, $M_{0.025}$ the most dense and $M_{B I O-P O R E}$ was the one used by Samsó and García $(2013 \mathrm{a}, \mathrm{b})$. $M_{B I O-P O R E}$ was the only mesh with a predefined numbers of elements at boundaries 3 (20 elements), 4 (550 elements) and 5 (7 elements), which were reckoned as the most critical ones numerically (large concentration gradients).

Simulated effluent concentrations of COD (sum of $S_{F}, S_{A}, S_{I}, X_{S m}$ and $\left.X_{I m}\right)$ and $S_{N H}$, as well as the simulation time were recorded for all different 
meshes. Although the simulated effluent concentrations of the rest of model components could have also been studied, only COD and $S_{N H}$ were used for the sake of brevity and because these are the two most widely used water quality indicators. The Sum of Squared Errors (SSE) for the effluent COD and $S_{N H}$ curves for all different meshes were calculated using the coarser mesh $\left(M_{0.1}\right)$ as a reference, to showcase the progressive accuracy gains with increasing mesh densities. The optimal mesh corresponds to that after which any further increments on the number of elements does not produce notable improvements on the numerical accuracy of the solution (SSE remains fairly constant). Moreover, for evident practical reasons, the optimal mesh is also that with the shortest computational cost/time.

\subsection{Parameter sensitivity}

The sensitivity of $M_{\text {bio_max }}$ and $M_{c a p}$ was studied by giving three different values to each of the two parameters (Table 6) and running a different simulation for each different pair (9 simulations in total) (Table 7). The reason for selection the values of Table 6 are discussed later in the text. Notice that the range of variability of $M_{c a p}$ was smaller than that of $M_{\text {bio_max }}$. In the first case, the highest value of $M_{c a p}$ was 3 times the smallest, whereas for $M_{\text {bio_max }}$ the highest was 5 times the smallest.

The sensitivity of the two parameters was determined qualitatively by comparing the effluent concentrations of COD among them with the 9 different parameter pairs. The same is done for the simulated effluent concentrations of $S_{N H}$. A qualitative comparison was made between the effluent concentrations of COD and $S_{N H}$ obtained with each parameter pair. 
The mesh used to execute all these processes was the optimum mesh obtained in the previous step (Section 2.2).

\subsection{Launching simulations and hardware specifications}

In this work two different computers were used. For the mesh optimization procedure, a desktop PC was used. This computer features an Intel ${ }^{\circledR}$ Xenon ${ }^{\circledR}$ E5-1620 processor with 4 cores (8 threads) running at a frequency of $3600 \mathrm{GHz}$ and $16 \mathrm{~GB}$ of RAM memory. The Linux kernel and COMSOL Multiphysics ${ }^{T M}$ versions installed on this computer were 3.2.0-56 and v4.3b, respectively.

On the other hand, for the sensitivity analysis the cluster functionalities of COMSOL Multiphysics ${ }^{T M}$ were used to run several simulations in parallel on a high-end multi-processor computer. This computer consisted of 4 CPUs AMD Opteron ${ }^{T M} 6140$ with 8 cores each $(2.6 \mathrm{GHz})$, a total of $64 \mathrm{~GB}$ of RAM memory and run Linux Kernel 2.6.38. The COMSOl Multiphysics ${ }^{T M}$ version installed in this machine was v4.2a. Since this machine was shared with other researchers, only 3 parallel simulations (using $4 \mathrm{CPU}$ cores each) were launched at a time (see Figure 2). Therefore only 12 cores, out of the 32 available, were utilized. A bash script was used to automatically launch each different batch of 3 parallel simulations without any intervention.

\section{Results and discussion}

\subsection{Mesh optimization}

In the current study the focus was not on how well or bad simulated effluent concentrations fit experimental data, since that discussion was already 
made in Samsó and García (2013a), but rather on the comparison of the simulation results obtained with different meshes. However, note that the poor fitting of the simulated effluent COD and $S_{N H}$ with experimental data at the beginning of all simulations (Figures 3 and 4), was due to the fact that initial bacteria and accumulated solids concentrations were underestimated. However, after around 70 days of simulated time, the fitting improved.

Figures 3 and 4 show that the effluent pollutant concentrations of COD and $S_{N H}$ obtained with the different meshes (Table 5) are visually different in some cases.

Finer FE meshes provide more accurate numerical results. Thus in our study, mesh $M_{0.025}$, with a maximum element size of $2,5 \mathrm{~cm}$ and a total number of 28884 elements is the one giving more accurate results. Despite even better results could have been obtained by further refining the mesh, the total simulation time of $M_{0.025}$ (16 hours and 18 minutes) was already seen as too large for practical reasons. Moreover, refining the mesh to such an extent would only make sense if field data, which is given as model input and later used to compare with simulated effluent concentrations, had been gathered in higher frequency. In fact, Figure 4 clearly shows that almost identical results were obtained for simulated effluent $S_{N H}$ concentrations with meshes $M_{B I O-P O R E}$ and $M_{0.03}$ which account for c.a. $30 \%$ less elements than $M_{0.025}$. That is also confirmed with the tendency of the SSE for $S_{N H}$ (Figure 6), which shows clear signs of stabilization already with meshes $M_{B I O-P O R E}$ and $M_{0.03}$. Therefore, further mesh refinements would not improve the description of the effluent $S_{N H}$ concentrations. In the case of COD (Figure 3), although the differences between the curves obtained with different meshes were higher 
than for $S_{N H}$, and the SSE still did not show signs of stabilization (Figure 5), the maximum difference of effluent COD concentrations obtained with meshes $M_{B I O-P O R E}$ and $M_{0.025}$ was lower than $15 \mathrm{mgCOD} \cdot L^{-1}$, which was only around $8 \%$ the maximum effluent COD concentration simulated with mesh $M_{0.025}$. Moreover, note that the reference mesh $M_{0.1}$ was already fine (1860 elements) and thus reaching SSE stability is more difficult than if a coarser mesh had been used as a reference to calculate SSE.

Table 8 shows that, in general, the simulation time increased with increasing mesh densities. $M_{B I O-P O R E}$ was the exception, and although it had 213 less elements than $M_{0.03}$ the former took 25 minutes more than the later to reach the final solution (see Table 8). The most likely reason for that is that the mesh element quality of $M_{B I O-P O R E}$ was lower than that of $M_{0.03}$ and thus the solver algorithm required a few more iterations at every time step to reach a solution. In fact, $M_{B I O-P O R E}$ was the one with the second largest maximum element size $(0.05 \mathrm{~cm})$, only after $M_{0.1}$, but in contrast it was the mesh with the highest elements densities in boundaries 3,4 and 5 , which were the ones accounting for the highest concentration gradients. The relation between number of elements and simulated time can also be observed in Figures 5 and 6 , and shows that a linear relationship $\left(R^{2}=0.97\right)$ exists between the two.

According to these results, the mesh with a better compromise between numerical accuracy and simulation time was $M_{0.03}$. The results obtained with mesh $M_{B I O-P O R E}$ were almost as good as those obtained with $M_{0.03}$ (see Figures 3, 4, 5 and 6), and since mesh $M_{B I O-P O R E}$ had already been used successfully in a previous work (Samsó and García, 2013a), it was chosen 
as the one to be used for the sensitivity analysis.

\subsection{Parameter sensitivity}

Despite BIO_PORE includes more than 50 parameters, only the sensitivity of $M_{\text {bio_max }}$ and $M_{c a p}$ was analysed because they are two new additions to the formulation of CWM1. Moreover, the sensitivity of the different parameters of CWM1 has already been studied in other works (Mburu et al., 2012). Note that the type of analysis carried out in this work is a local sensitivity analysis, which only addresses sensitivity relative to the point estimates chosen and not for the entire parameter distribution.

The first parenthesis of Eq. 4 (involving $M_{\text {bio_max }}$ ) limits the maximum concentration of bacteria that each pore of the granular media can hold (carrying capacity) by stopping the growth of bacteria once $M_{b i o}$ reaches the value of $M_{\text {bio_max }}$. The second parenthesis works in the same way, but $M_{c a p}$ corresponds to the maximum amount of particulate solids $\left(X_{S f}\right.$ and $\left.X_{I f}\right)$ porosity can hold, and bacterial growth stops once $M_{X_{I f}}=M_{c a p}$.

The values given to parameters $M_{\text {bio_max }}$ were chosen based on our previous experiences with the BIO_PORE model, since no literature values for these parameters exist for CWs. In fact the intermediate value of this parameter used in the current work was that obtained from the calibration of the model in Samsó and García (2013a), and the other two were chosen to be at a sound distance from the first. On the other hand, the amount of accumulated solids in horizontal subsurface flow CWs presents a great variability depending on the COD and TSS loading rates and on the turn-over rates. Measurements carried out by Caselles-Osorio et al. (2007) in 6 fullscale horizontal subsurface flow CWs showed that accumulated solids ranged 
from as low as $2.3 \mathrm{kgVS} \cdot \mathrm{m}^{-2}$ up to $57.3 \mathrm{kgVS} \cdot \mathrm{m}^{-2}$ (between around 6 and $162 \mathrm{kgCOD} \cdot \mathrm{m}^{-3}$, considering an average wetland depth of $0.5 \mathrm{~m}$ and that $1 g V S \approx 1.42 g C O D$ (Samsó and García, 2014a)). In this study we selected the values of $M_{\text {cap }}$ to be in the lower part of that range, since the gravel size of the pilot system was quite fine $\left(D_{60}=3.5 \mathrm{~mm}\right.$ and $\left.C_{u}=1.7\right)$.

Results indicate that $M_{b i o_{\text {max }}}$ and $M_{c a p}$ are both very sensitive parameters since they had a large impact on the simulated concentrations of COD (Figure 7 ) and $S_{N H}$ (Figure 8). At the beginning of all simulations, effluent concentrations obtained with the different pairs of $M_{\text {bio_max }}$ and $M_{\text {cap }}$ were very similar, and it was not until around simulated day 60 that they started diverging. Figures 7 and 8 show that both for COD and $S_{N H}$ the most sensitive parameter was $M_{\text {cap }}$, and the higher its value, and thus the higher the capacity of porosity to retain particulate solids $\left(X_{I f}\right.$ and $\left.X_{S f}\right)$, the lower the effluent concentrations of the two pollutants. A possible reasoning for this behaviour is that for high values of $M_{\text {cap }}$ the amount of slowly biodegradable particulate COD $\left(X_{S f}\right)$ that can be reached in the granular media is much higher than that the maximum bacteria biomass present in the same location (which is limited by the value of $M_{\text {bio_max }}$ ) can biodegrade, and so they accumulate. Therefore this accumulated organic matter, which also contains a fraction of organic nitrogen, is retained within the system and does not add to the concentrations of COD and $S_{N H}$ measured at the outlet.

On the other hand, although perturbations of the $M_{\text {bio_max }}$ value produced observable changes in the effluent COD and $S_{N H}$ concentrations, these changes were smaller than those produced by changing the value of $M_{\text {cap }}$. Regarding the effluent COD concentrations (Figure 7), for $M_{\text {cap }}=15 \mathrm{kgVS} \cdot \mathrm{m}^{-3}$ 
and $M_{\text {cap }}=10 \mathrm{kgVS} \cdot \mathrm{m}^{-3}$, the higher the value of $M_{\text {bio_max }}$ the higher the effluent concentrations of COD. This can be explained by the fact that the higher the maximum concentrations of biomass in a specific point of the granular media, the larger proportion of the accumulated $X_{S f}$ can be hydrolysed and thus released through the outlet (in the form of $S_{F}, S_{A}, S_{I}$ and $S_{N H}$ ) increasing the effluent concentrations of COD and $S_{N H}$. On the contrary, for $M_{c a p}=5 \mathrm{kgVS} \cdot \mathrm{m}^{-3}$, the tendency is different and the effluent concentrations are higher for $M_{\text {bio_max }}=0.3 \mathrm{kgVS} \cdot \mathrm{m}^{-3}$, intermediate for $M_{\text {bio_max }}=0.1 \mathrm{Kg} \cdot \mathrm{m}^{-3}$ and the lowest for $M_{\text {bio_max }}=0.5 \mathrm{KgVS} \cdot \mathrm{m}^{-3}$. Therefore no clear pattern can be extracted for $M_{\text {bio_max }}$ when the values of $M_{\text {cap }}$ are relatively small.

Regarding $S_{N H}$ (Figure 8), for $M_{c a p}=15 \mathrm{kgVS} \cdot \mathrm{m}^{-3}$, the effluent concentrations of this component are almost the same regardless of the value of $M_{\text {bio_max }}$. For the intermediate value of $M_{\text {cap }}\left(10 \mathrm{kgVS} \cdot \mathrm{m}^{-3}\right)$, $M_{\text {bio_max }}=0.5 \mathrm{kgVS} \cdot \mathrm{m}^{-3}$ gives the highest effluent concentration, while for $M_{\text {bio_max }}=0.3 \mathrm{kgVS} \cdot \mathrm{m}^{-3}$ and $M_{\text {bio_max }}=0.1 \mathrm{kgVS} \cdot \mathrm{m}^{-3}$ the effluent concentrations are almost identical. For the lowest value of $M_{c a p}$ $\left(5 \mathrm{kgVS} \cdot \mathrm{m}^{-3}\right)$ there are also differences between the curves, but in this case $M_{\text {bio_max }}=0.1 \mathrm{kgVS} \cdot \mathrm{m}^{-3}$ gives the lowest effluent concentrations of $S_{N H}$ while $M_{\text {bio_max }}=0.5 \mathrm{kgVS} \cdot \mathrm{m}^{-3}$ and $M_{\text {bio_max }}=0.3 \mathrm{kgVS} \cdot \mathrm{m}^{-3}$ give almost the same results.

Therefore, contrarily to what happened for $M_{\text {cap }}$, for $M_{\text {bio_max }}$ although some patterns can be detected for the effluent COD concentrations, there is not a clear distinguishable tendency regarding the effluent concentrations of $S_{N H}$ obtained with the different values of this parameter. However, the 
higher the value of $M_{\text {cap }}$, the larger the difference between the effluent concentrations obtained with the different values o $M_{\text {bio_max }}$.

\section{Conclusions}

In this work we performed a mesh optimization procedure in order to reduce the simulation time (while maintaining similar numerical accuracy) for subsequent simulations, and we also performed a local sensitivity analysis of parameters $M_{c a p}$ and $M_{\text {bio_max }}$.

Results of the mesh optimization procedure indicated that for homogeneous meshes, a positive linear relationship existed between the number of elements and simulated time. The best compromise between numerical accuracy and computational cost was obtained with meshes $M_{0.03}$ and $M_{B I O-P O R E}$. Therefore $M_{B I O-P O R E}$ was selected as the optimal mesh to carry out the sensitivity analysis.

Despite the range of values given to $M_{c a p}$ was smaller than that given to $M_{\text {bio_max }}$, the former parameter proved to be the most sensitive one, and the higher its value the lower the simulated effluent concentrations of COD and $S_{N H}$ of the wetland. This was due to the fact that for larger values of $M_{c a p}$, more slowly biodegradable solids can accumulate in a specific point, and if there is not enough bacteria to hydrolyse them, they are not released and thus the effluent concentrations of COD and $S_{N H}$ does not increase.

On the other hand, from the values given to $M_{\text {bio_max }}$ no clear recognisable pattern on the effluent concentrations of COD and $S_{N H}$ could be observed. 


\section{Acknowledgements}

Roger Samsó acknowledges the scholarship provided by the Universitat Politècnica de Catalunya (UPC).

\section{References}

Batstone, D.J., Keller, J., Angelidaki, I., Kalyuzhny, S.V., Pavlostathis, S.G., Rozzi, A., Sanders, W.T.M., Siegrist, H., Vavilin, V.A., 2002. Anaerobic digestion model No. 1 (ADM1). IWA Publishing.

Brovelli, A., Malaguerra, F., Barry, D., 2009. Bioclogging in porous media: Model development and sensitivity to initial conditions. Environmental Modelling \& Software 24, 611-626. doi:10.1016/j.envsoft.2008.10.001.

Caselles-Osorio, A., Puigagut, J., Segú, E., Vaello, N., Granés, F., García, D., García, J., 2007. Solids accumulation in six full-scale subsurface flow constructed wetlands. Water research 41, 1388-98. doi:10.1016/j.watres.2006.12.019.

Clement, T.P., Sun, Y., Hooker, B., Peterser, J., 1998. Modeling Multispecies Reactive Transport in Ground Water. Groundwater Monitoring and Remediation 18, 79-92.

García, J., Aguirre, P., Mujeriego, R., Huang, Y., Ortiz, L., Bayona, J.M., 2004b. Initial contaminant removal performance factors in horizontal flow reed beds used for treating urban wastewater. Water research 38, 1669-78. doi:10.1016/j.watres.2004.01.011. 
García, J., Chiva, J., Aguirre, P., Alvarez, E., Sierra, J., Mujeriego, R., 2004a. Hydraulic behaviour of horizontal subsurface flow constructed wetlands with different aspect ratio and granular medium size. Ecological Engineering 23, 177-187. doi:10.1016/j.ecoleng.2004.09.002.

García, J., Rousseau, D.P.L., Morató, J., Lesage, E., Matamoros, V., Bayona, J.M., 2010. Contaminant Removal Processes in Subsurface-Flow Constructed Wetlands: A Review. Critical Reviews in Environmental Science and Technology 40, 561-661. doi:10.1080/10643380802471076.

Henze, M., on Mathematical Modelling for Design, I.W.A.T.G., of Biological Wastewater Treatment, O., 2000. Activated Sludge Models ASM1, ASM2, ASM2d and ASM3. Eighteenth century collections online, IWA Publishing.

Langergraber, G., Rousseau, D.P.L., García, J., Mena, J., 2009. CWM1: a general model to describe biokinetic processes in subsurface flow constructed wetlands. Water science and technology : a journal of the International Association on Water Pollution Research 59, 1687-1697. doi:10.2166/wst.2009.131.

Mburu, N., Sánchez-Ramos, D., Rousseau, D.P., van Bruggen, J.J., Thumbi, G., Stein, O.R., Hook, P.B., Lens, P.N., 2012. Simulation of carbon, nitrogen and sulphur conversion in batch-operated experimental wetland mesocosms. Ecological Engineering 42, 304-315. doi:10.1016/j.ecoleng.2012.02.003.

Meyer, D., Chazarenc, F., Claveau-Mallet, D., Dittmer, U., Forquet, N., Molle, P., Morvannou, A., Pálfy, T., Petitjean, A., Rizzo, A., Samsó, R., 
Scholz, M., Soric, A., Langergraber, G., 2014. Modelling constructed wetlands: scopes and aims - a review. Ecological Engineering (accepted) .

Monod, J., 1949. The growth of bacterial cultures. Cultures .

Puigagut, J., Villasenor, J., Salas, J., Bécares, E., García, J., 2007. Subsurface-flow constructed wetlands in Spain for the sanitation of small communities: A comparative study. Ecological Engineering 30, 312-319. doi:10.1016/j.ecoleng.2007.04.005.

Samsó, R., García, J., 2013a. BIO_PORE, a mathematical model to simulate biofilm growth and water quality improvement in porous media: Application and calibration for constructed wetlands. Ecological Engineering 54, 116-127. doi:10.1016/j.ecoleng.2013.01.021.

Samsó, R., García, J., 2013b. Bacteria distribution and dynamics in constructed wetlands based on modelling results. Science of The Total Environment 461-462, 430-440. doi:10.1016/j.scitotenv.2013.04.073.

Samsó, R., García, J., 2014a. The Cartridge Theory: A description of the functioning of horizontal subsurface flow constructed wetlands for wastewater treatment, based on modelling results. Science of The Total Environment 473-474, 651-658. doi:10.1016/j.scitotenv.2013.12.070.

Samsó, R., Meyer, D., García, J., 2014b. Subsurface flow constructed wetlands models: review and prospects, in: Vymazal, J. (Ed.), The Role of Natural and Constructed Wetlands in Nutrient Cycling and Retention on the Landscape. Springer, Dordrecht, The Netherlands (in press). 
${ }_{432}$ Tyroller, L., Rousseau, D.P.L., Santa, S., García, J., 2010. Applica433 tion of the gas tracer method for measuring oxygen transfer rates in 434 subsurface flow constructed wetlands. Water research 44, 4217-25. 435 doi:10.1016/j.watres.2010.05.027. 
Table 1: Description of the components considered in BIO_PORE model . $S_{i}$ are dissolved species (all in the aqueous phase by definition) and $X_{i}$ are particulate species (either in aqueous or solid phase).

\begin{tabular}{|l|l|l|l|}
\hline Componenet & Description & Unit & Phase \\
\hline$S_{O}$ & Dissolved oxygen & $m g C O D \cdot L^{-1}$ & Aqueous \\
\hline$S_{F}$ & Soluble fermentable COD & $m g C O D \cdot L^{-1}$ & Aqueous \\
\hline$S_{A}$ & $\begin{array}{l}\text { Fermentation products as acetate } \\
\text { as COD }\end{array}$ & $m g C O D \cdot L^{-1}$ & Aqueous \\
\hline$S_{I}$ & Inert soluble COD & $m g C O D \cdot L^{-1}$ & Aqueous \\
\hline$X_{S m}$ & $\begin{array}{l}\text { Aqueous slowly biodegradable } \\
\text { particulate COD }\end{array}$ & $m g C O D \cdot L^{-1}$ & Aqueous \\
\hline$X_{S f}$ & $\begin{array}{l}\text { Solid slowly biodegradable partic- } \\
\text { ulate COD }\end{array}$ & $m g C O D \cdot L^{-1}$ & Solid \\
\hline$X_{I m}$ & Aqueous inert particulate COD & $m g C O D \cdot L^{-1}$ & Aqueous \\
\hline$X_{I f}$ & Solid inert particulate COD & $m g C O D \cdot L^{-1}$ & Solid \\
\hline$S_{N O}$ & Nitrite and nitrate nitrogen & $m g N \cdot L^{-1}$ & Aqueous \\
\hline$S_{N H}$ & $\begin{array}{l}\text { Ammonium and ammonia nitro- } \\
\text { gen }\end{array}$ & $m g N \cdot L^{-1}$ & Aqueous \\
\hline$S_{S O 4}$ & Sulphate sulphur & $m g S \cdot L^{-1}$ & Aqueous \\
\hline$S_{H 2 S}$ & Dihydrogensulphide sulphur & $m g S \cdot L^{-1}$ & Aqueous \\
\hline
\end{tabular}


Table 2: Functional bacterial groups considered in BIO_PORE. Bacteria concentrations are given in units of COD $\left(m g C O D \cdot L^{-1}\right)$.

\begin{tabular}{|l|l|l|}
\hline Component & Description & Phase \\
\hline$X_{H}$ & Heterotrophic bacteria & Solid \\
\hline$X_{A}$ & Autotrophic nitrifying bacteria & Solid \\
\hline$X_{F B}$ & Fermenting bacteria & Solid \\
\hline$X_{A M B}$ & Acetotrophic methanogenic bacteria & Solid \\
\hline$X_{A S R B}$ & Acetotrophic sulphate reducing bacteria & Solid \\
\hline$X_{S O B}$ & Sulphide oxidising bacteria & Solid \\
\hline
\end{tabular}


Table 3: Processes rates in $m g \cdot d^{-1}$ (adapted from Langergraber et al. (2009)).

\section{j Process}

1 Hydrolysis $X_{S f}$

2 Aerobic growth of $X_{H}$ on $S_{F}$

3 Anoxic growth of $X_{H}$ on $S_{F}$

4 Aerobic growth of $X_{H}$ on $S_{A}$

5 Anoxic growth of $X_{H}$ on $S_{A}$

6 Lysis of $X_{H}$

7 Aerobic growth of $X_{A}$ on $S_{N H}$

8 Lysis of $X_{A}$

9 Growth of $X_{F B}$

10 Lysis of $X_{F B}$

11 Growth of $X_{A M B}$

12 Lysis of $X_{A M B}$

13 Growth of $X_{A S R B}$

14 Lysis of $X_{X A S R B}$

15 Aerobic growth of $X_{S O B}$ on $S_{H 2 S}$

16 Anoxic growth of $X_{S O B}$ on $S_{H 2 S}$

17 Lysis of $X_{S O B}$

\section{Process rate $\rho_{j}$}

$k_{h}\left[\frac{\frac{X_{S f}}{X_{H}+X_{F B}}}{K_{X}\left(\frac{X_{S f}}{X_{H}+X_{F B}}\right)}\right]\left(X_{H}+\eta_{h} X_{F B}\right)$

$\mu_{H} \cdot f_{G L}\left(\frac{S_{F}}{K_{S F H}+S_{F}}\right)\left(\frac{S_{F}}{S_{F}+S_{A}}\right)\left(\frac{S_{O}}{K_{S O H}+S_{O}}\right)\left(\frac{S_{N H}}{K_{S N H H}+S_{N H}}\right)\left(\frac{K_{S H 2 S H}}{K_{S H} 2 S H+S_{H 2 S}}\right) X_{H}$

$\eta_{g} \cdot \mu_{H} \cdot f_{G L}\left(\frac{S_{F}}{K_{S F H}+S_{F}}\right)\left(\frac{S_{F}}{S_{F}+S_{A}}\right)\left(\frac{K_{S O H}}{K_{S O H}+S_{O}}\right)\left(\frac{S_{N O}}{K_{S N O H}+S_{N O}}\right)\left(\frac{S_{N H}}{K_{S N H H}+S_{N H}}\right)\left(\frac{K_{S H 2 S H}}{K_{S H 2 S H}+S_{H 2 S}}\right) X_{H}$

$\mu_{H} \cdot f_{G L}\left(\frac{S_{A}}{K_{S A H}+S_{A}}\right)\left(\frac{S_{A}}{S_{F}+S_{A}}\right)\left(\frac{S_{O}}{K_{S O H}+S_{O}}\right)\left(\frac{S_{N H}}{K_{S N H H}+S_{N H}}\right)\left(\frac{K_{S H 2 S H}}{K_{S H 2 S H}+S_{H 2 S}}\right) X_{H}$

$\eta_{g} \mu_{H} \cdot f_{G L}\left(\frac{S_{A}}{K_{S A H}+S_{A}}\right)\left(\frac{S_{A}}{S_{F}+S_{A}}\right)\left(\frac{K_{S O H}}{K_{S O H}+S_{O}}\right)\left(\frac{S_{N O}}{K_{S N O H}+S_{N O}}\right)\left(\frac{S_{N H}}{K_{S N H}+S_{N H}}\right)\left(\frac{K_{S H 2 S H}}{K_{S H} 2 S H+S_{H 2 S}}\right) X_{H}$

$b_{X} X_{H}$

$\mu_{A} \cdot f_{G L}\left(\frac{S_{N H}}{K_{S N H A}+S_{N H}}\right)\left(\frac{S_{O}}{K_{S O A}+S_{O}}\right)\left(\frac{K_{S H 2 S A}}{K_{S H 2 S A}+S_{H 2 S}}\right) X_{A}$

$b_{A} X_{A}$

$\mu_{F B} \cdot f_{G L}\left(\frac{S_{F}}{K_{S F F B}+S_{F}}\right)\left(\frac{K_{S H 2 S F B}}{K_{S H 2 S F B}+S_{H 2 S}}\right)\left(\frac{K_{S O F B}}{K_{S O F B}+S_{O}}\right)\left(\frac{K_{S N O F B}}{K_{S N O F B}+S_{N O}}\right)\left(\frac{S_{N H}}{K_{S N H F B}+S_{N H}}\right) X_{F B}$

$b_{F B} X_{F B}$

$\mu_{A M B} \cdot f_{G L}\left(\frac{S_{A}}{K_{S A M B}+S_{A}}\right)\left(\frac{K_{S H 2 S A M B}}{K_{S H 2 S A M B}+S_{H 2 S}}\right)\left(\frac{K_{S O A M B}}{K_{S O A M B}+S_{O}}\right)\left(\frac{K_{S N O A M B}}{K_{S N O A M B}+S_{N O}}\right)\left(\frac{S_{N H}}{K_{S N H A M B}+S_{N H}}\right) X_{A M B}$ $b_{A M B} X_{A M B}$

$\mu_{A S R B} \cdot f_{G L}\left(\frac{S A}{K_{S A A S R B}+S_{A}}\right)\left(\frac{S_{S O 4}}{K_{S O 4 A S R B}+S_{S O 4}}\right)\left(\frac{K_{S H 2 S A S R B}}{K_{S H 2 S A S R B}+S_{H 2 S}}\right)\left(\frac{K_{S O A S R B}}{K_{S O A S R B}+S_{O}}\right)\left(\frac{K_{S N O A S R B}}{K_{S N O A S R B}+S_{N O}}\right)$ $\left(\frac{S_{N H}}{K_{S N H A S R B}+S_{N H}}\right) X_{A S R B}$

$b_{A S R B} X_{A S R B}$

$\mu_{S O B} \cdot f_{G L}\left(\frac{S_{H 2 S}}{K_{S H 2 S S O B}+S_{H 2 S}}\right)\left(\frac{S_{O}}{K_{S O S O B}+S_{O}}\right)\left(\frac{S_{N H}}{K_{S N H S O B}+S_{N H}}\right) X_{S O B}$

$\mu_{S O B} \cdot f_{G L} \cdot \eta_{S O B}\left(\frac{S_{H 2 S}}{K_{S H 2 S S O B}+S_{H 2 S}}\right)\left(\frac{S_{N O}}{K_{S N O S O B}+S_{N O}}\right)\left(\frac{K_{S O S O B}}{K_{S O S O B}+S_{O}}\right)\left(\frac{S_{N H}}{K_{S N H S O B}+S_{N H}}\right) X_{S O B}$

$b_{S O B} X_{S O B}$ 
Table 4: Values of the hydraulic and hydrodynamic parameters of the granular media.

\begin{tabular}{|c|l|c|c|}
\hline Parameter & Description & Unit & Value \\
\hline$\alpha_{L}$ & Longitudinal dispersivity & $\mathrm{m}$ & 0.05 \\
\hline$\alpha_{T}$ & Transverse dispersivity & $\mathrm{m}$ & 0.005 \\
\hline$K$ & Hydraulic conductivity & $m \cdot d^{-1}$ & 50 \\
\hline
\end{tabular}


Table 5: Meshes used in the mesh optimization procedure.

\begin{tabular}{|l|c|c|}
\hline Mesh & Maximum element size (m) & Number of elements \\
\hline$M_{0.1}$ & 0.1 & 1860 \\
\hline$M_{0.04}$ & 0.04 & 11446 \\
\hline$M_{\text {BIO-PORE }}$ & $0.05^{a}$ & 19851 \\
\hline$M_{0.03}$ & 0.03 & 20064 \\
\hline$M_{0.025}$ & 0.025 & 28884 \\
\hline
\end{tabular}

${ }^{a}$ Note that $M_{B I O-P O R E}$ was built with a maximum element size of $0.05 \mathrm{~m}$ but fixing the number of elements at boundaries 3 (20 elements), 4 (550 elements) and 5 (7 elements), and its total number of elements is very similar to that of $M_{0.03}$. 
Table 6: Values for $M_{c a p}$ and $M_{\text {bio_max }}$.

\begin{tabular}{|l|c|c|}
\hline Value & $M_{\text {cap }}\left(\mathrm{kgVS} \cdot \mathrm{m}^{-3}\right)$ & $M_{\text {bio_max }}\left(\mathrm{kgVS} \cdot \mathrm{m}^{-3}\right)$ \\
\hline Minimum & 3 & 0.1 \\
\hline Intermediate & 5 & 0.3 \\
\hline Maximum & 15 & 0.5 \\
\hline
\end{tabular}


Table 7: Combinations of $M_{c a p}$ and $M_{\text {bio_max }}$ values for the different simulations carried out for the local sensitivity analysis.

\begin{tabular}{|l|c|c|}
\hline Parameter & $M_{\text {cap }}\left(\mathrm{kgVS} \cdot \mathrm{m}^{-3}\right)$ & $M_{\text {bio_max }}\left(\mathrm{kg} V S \cdot \mathrm{m}^{-3}\right)$ \\
\hline$S_{1}$ & 15 & 0.5 \\
\hline$S_{2}$ & 15 & 0.3 \\
\hline$S_{3}$ & 15 & 0.1 \\
\hline$S_{4}$ & 10 & 0.5 \\
\hline$S_{5}$ & 10 & 0.3 \\
\hline$S_{6}$ & 10 & 0.1 \\
\hline$S_{7}$ & 5 & 0.5 \\
\hline$S_{8}$ & 5 & 0.3 \\
\hline$S_{9}$ & 5 & 0.1 \\
\hline
\end{tabular}


Table 8: Number of elements and simulation time for each of the meshes used for mesh optimization.

\begin{tabular}{|l|c|c|}
\hline Mesh & Number of triangular elements & Simulation time (hours) \\
\hline$M_{0.1}$ & 1860 & 1.04 \\
\hline$M_{0.04}$ & 11446 & 5.41 \\
\hline$M_{\text {BIO-PORE }}$ & 19851 & $9.96^{a}$ \\
\hline$M_{0.03}$ & 20064 & 9.53 \\
\hline$M_{0.025}$ & 28884 & 16.30 \\
\hline
\end{tabular}

${ }^{a}$ Notice that although $M_{B I O-P O R E}$ had fewer elements than $M_{0.03}$ its simulation time was slightly higher. Notice as well that $M_{B I O-P O R E}$ was the only one of the selected meshes with higher elements density in boundaries 3, 4 and 5 (see Figure 1). 


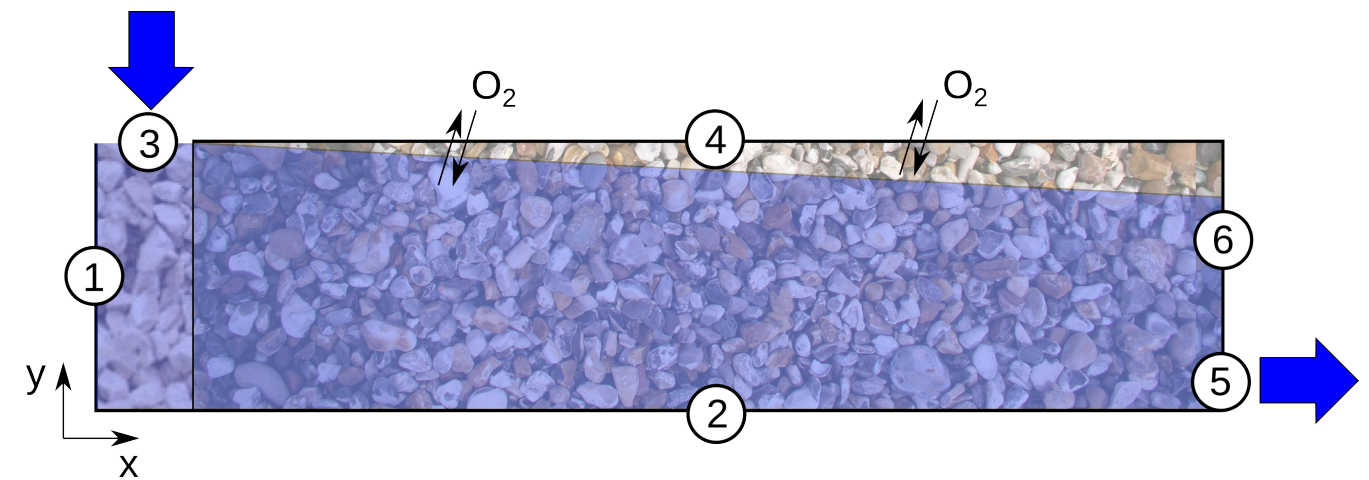

Figure 1: Model domain, representing a longitudinal section of wetland $C 2$ in García et al. (2004a), and numbers of the different boundaries (obtained from Samsó and García (2013a)). The numbers identify the different boundaries of the domain. Numbers 1 and 5 correspond to the inlet and outlet sections, respectively. 


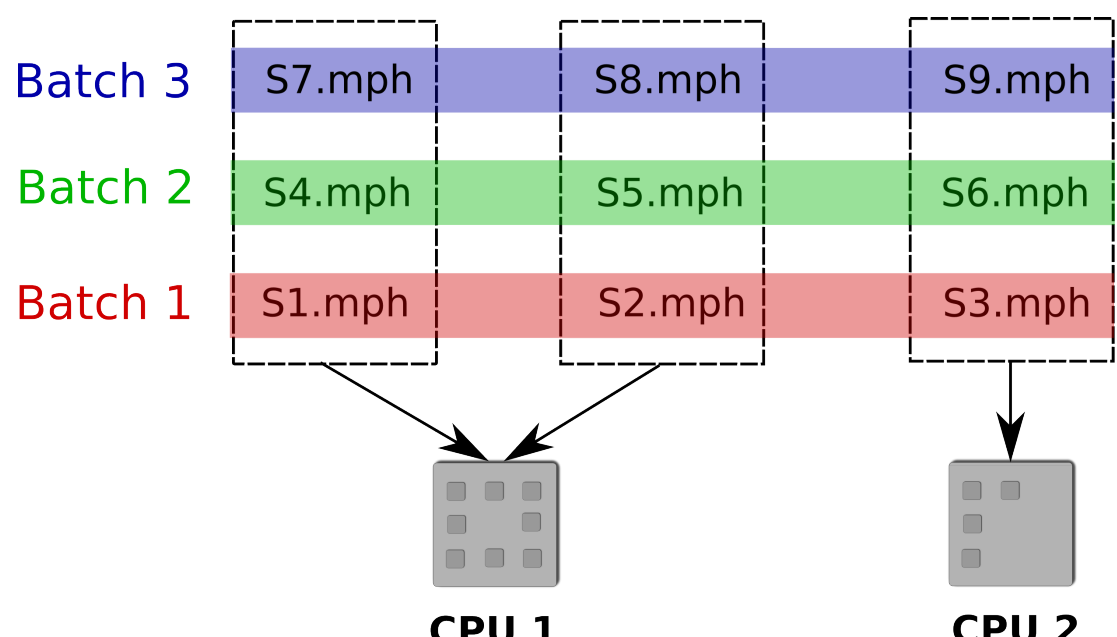

Figure 2: CPU and processor utilisation in the high-end multi-processor computer during the sensitivity analysis. Model files built in COMSOL Multiphysics ${ }^{T M}$ have $m p h$ extension. Three batches of 3 parallel simulations, each with a different $M_{c a p}-M_{\text {bio_max }}$ pair (see Table 7), were launched. Each simulation took up only 4 processor cores. All cores of CPU1 were used, while CPU2 was only loaded to a $50 \%$. 

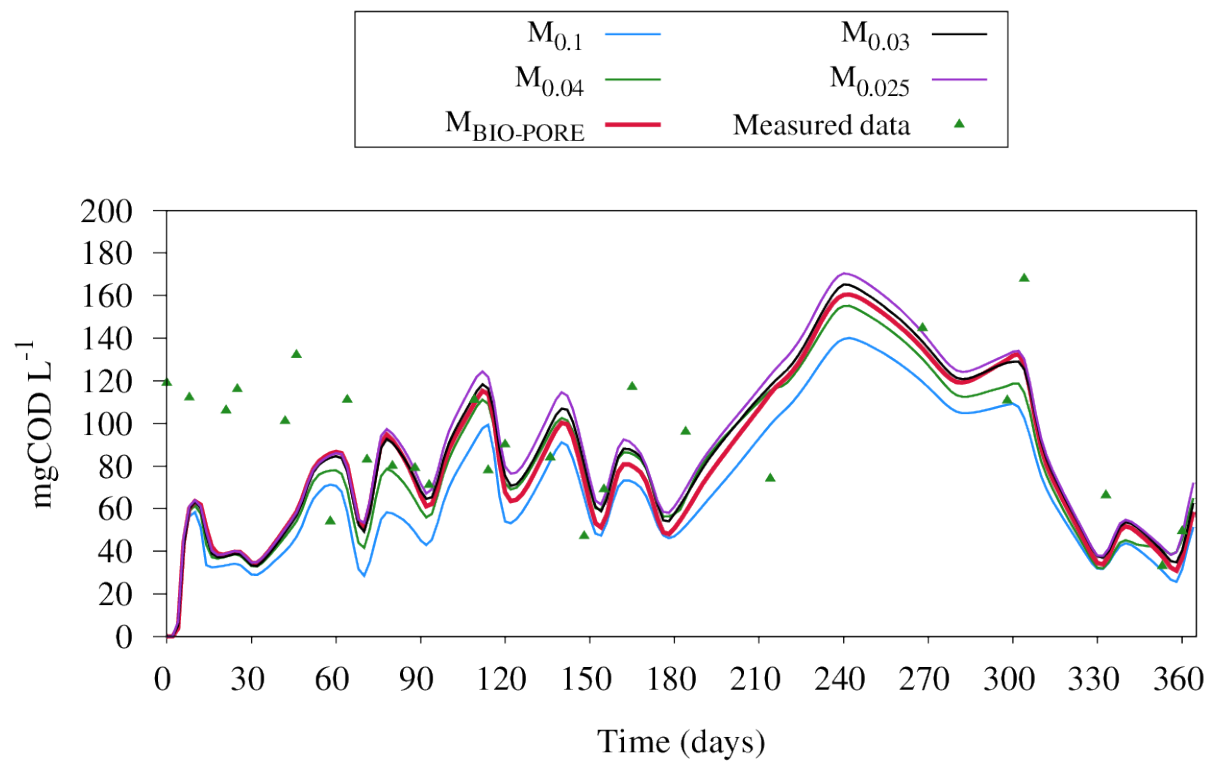

Figure 3: Simulated effluent COD concentrations obtained from the mesh optimization procedure with the meshes of Table 5 . 


\begin{tabular}{|rr|}
\hline $\mathrm{M}_{0.1}$ & $\mathrm{M}_{0.03}-$ \\
$\mathrm{M}_{0.04}$ & $\mathrm{M}_{0.025}-$ \\
$\mathrm{M}_{\text {BIO-PORE }}$ & - \\
Measured data
\end{tabular}

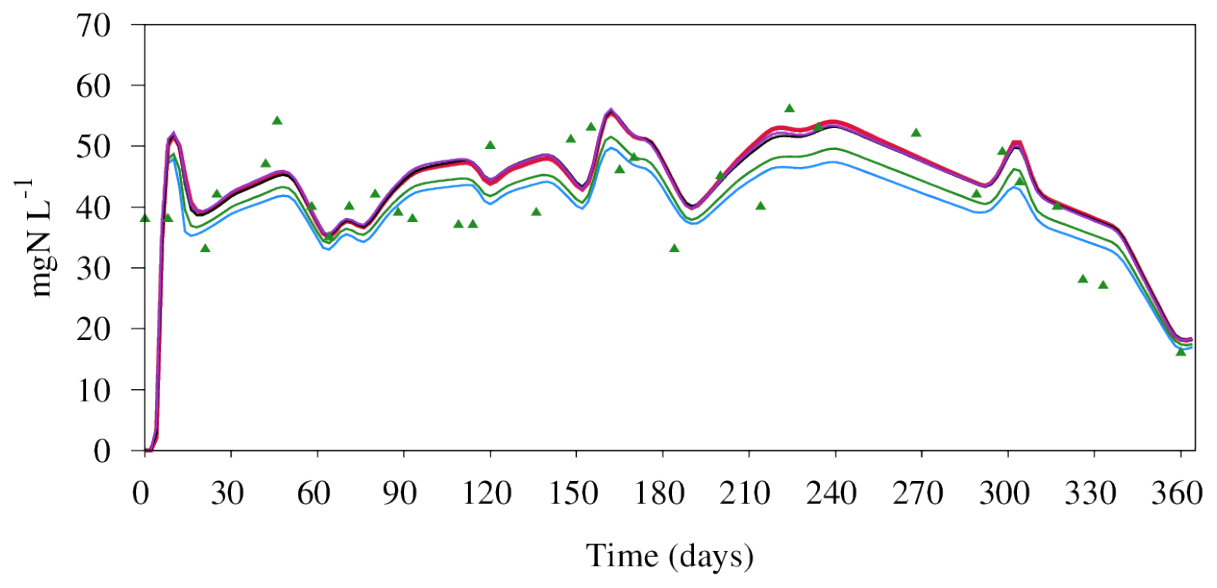

Figure 4: Simulated effluent $S_{N H}$ concentrations obtained from the mesh optimization procedure with the meshes of Table 5. 

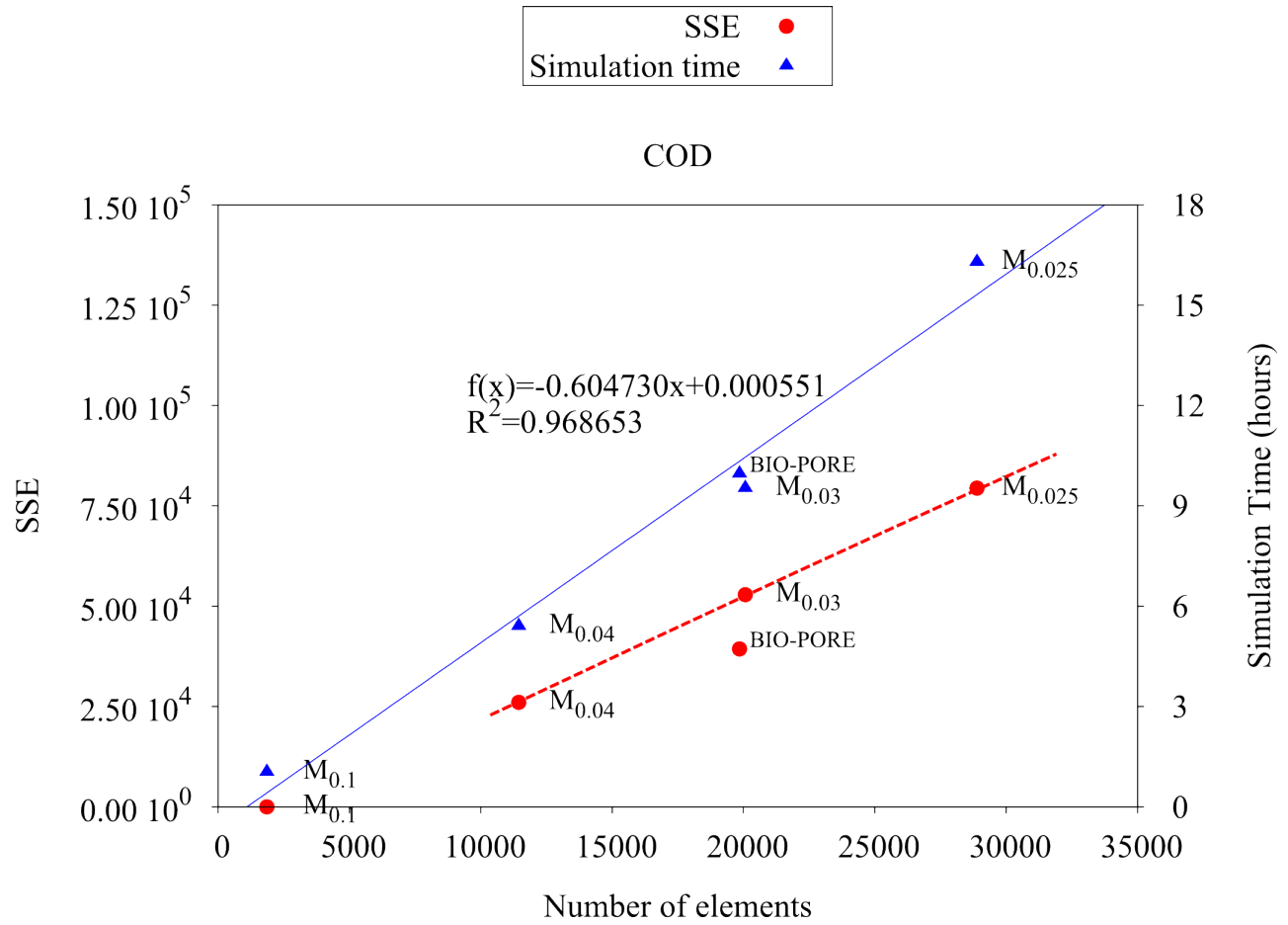

Figure 5: Sum of Squared Errors (SSE) (left y-axes) and simulation time (right y-axes) for the simulated effluent COD concentrations obtained with meshes of different elements density (see Table 6$)$. The blue line shows the positive linear relationship $\left(R^{2}=0.97\right)$ between the number of triangular elements of the mesh and the simulation time. The dotted red line was drawn to show that the SSE does not tend to a constant value with increasing number of elements. Notice that this line was drawn neglecting the SSE of $M_{B I O-P O R E}$ since this mesh was built with a pre-set number of elements in specific domain boundaries. Mesh $M_{0.1}$ was also neglected, since it was the reference mesh, from which all SSE plotted in this figure were calculated. 


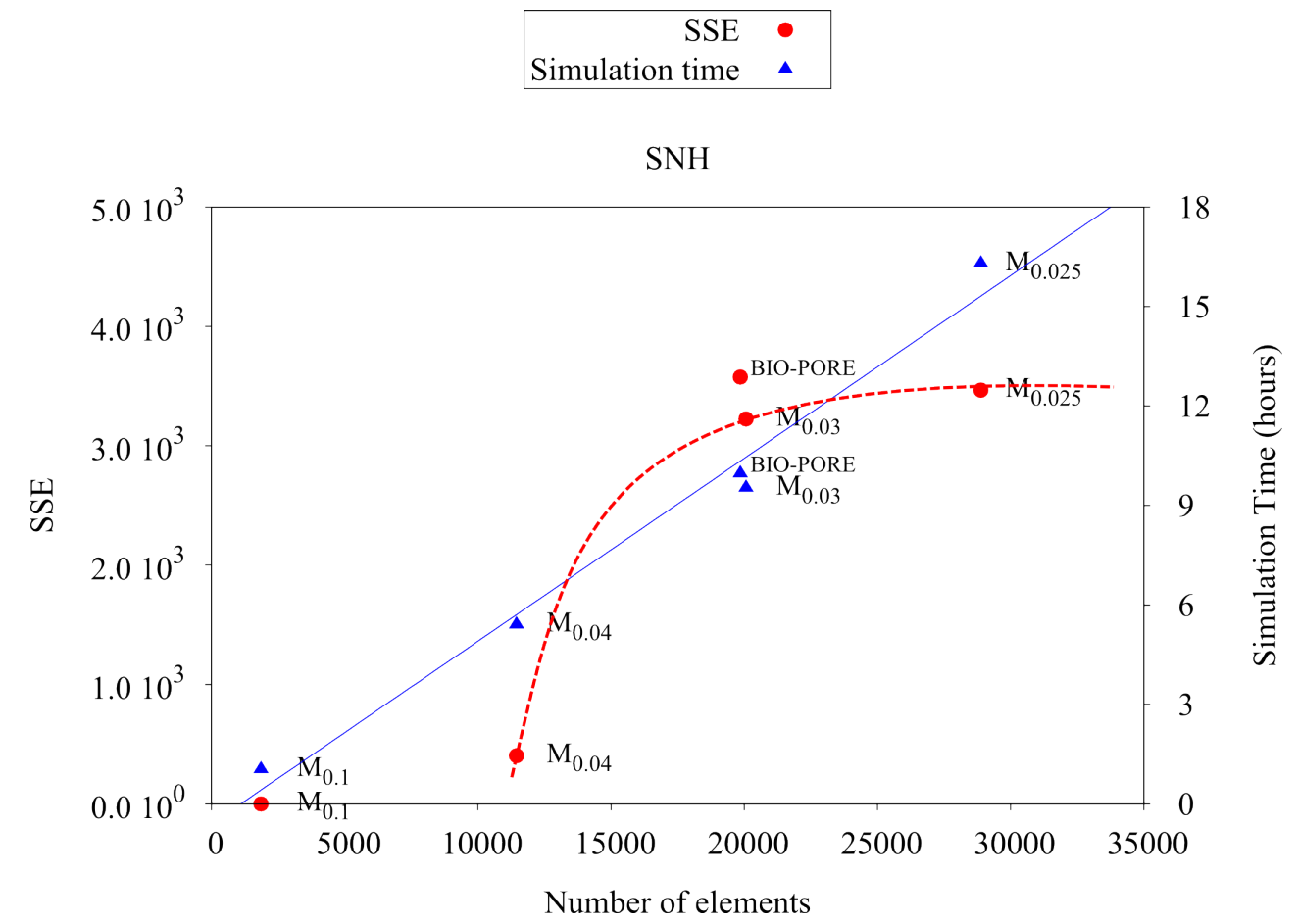

Figure 6: Sum of Squared Errors (SSE) (left y-axes) and simulation time (right y-axes) for the simulated effluent $S_{N H}$ concentrations obtained with meshes of different elements density (see Table 6 ). The $R^{2}$ of the linear regression of the Simulation time is the same as in Figure 5, since all data shown in both figures was obtained from the same simulations (each focusing on different model outputs). The dotted red line was drawn to show that for $S_{N H}$ the SSE tends to a constant value with increasing number of elements. As in the previous figure, the SSE of meshes $M_{B I O-P O R E}$ and $M_{0.1}$ were neglected to draw this line. 


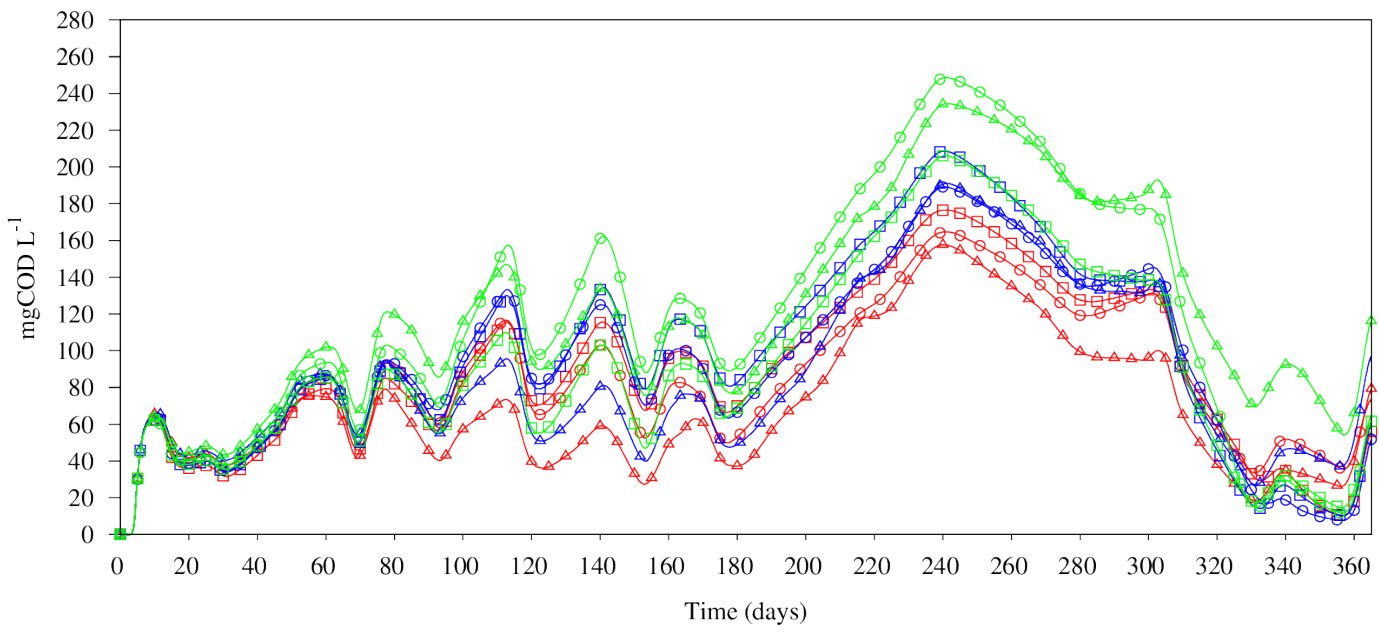

\begin{tabular}{|ll|}
\hline $\mathrm{S}_{15-0.5}-$ \\
$\mathrm{S}_{15-0.3}-$ \\
$\mathrm{S}_{15-0.1}-$ \\
$\mathrm{S}_{10-0.5}-\square$ \\
$\mathrm{S}_{10-0.3}-\bullet$ \\
$\mathrm{S}_{10-0.1}-\bullet$ \\
$\mathrm{S}_{5-0.5}-\square$ \\
$\mathrm{S}_{5-0.3}-\bullet$ \\
$\mathrm{S}_{5-0.1}-$ \\
\hline
\end{tabular}

Figure 7: Effluent COD concentrations obtained with the combinations of $M_{\text {bio_max }}$ and $M_{\text {cap }}$ shown in Table 7. 

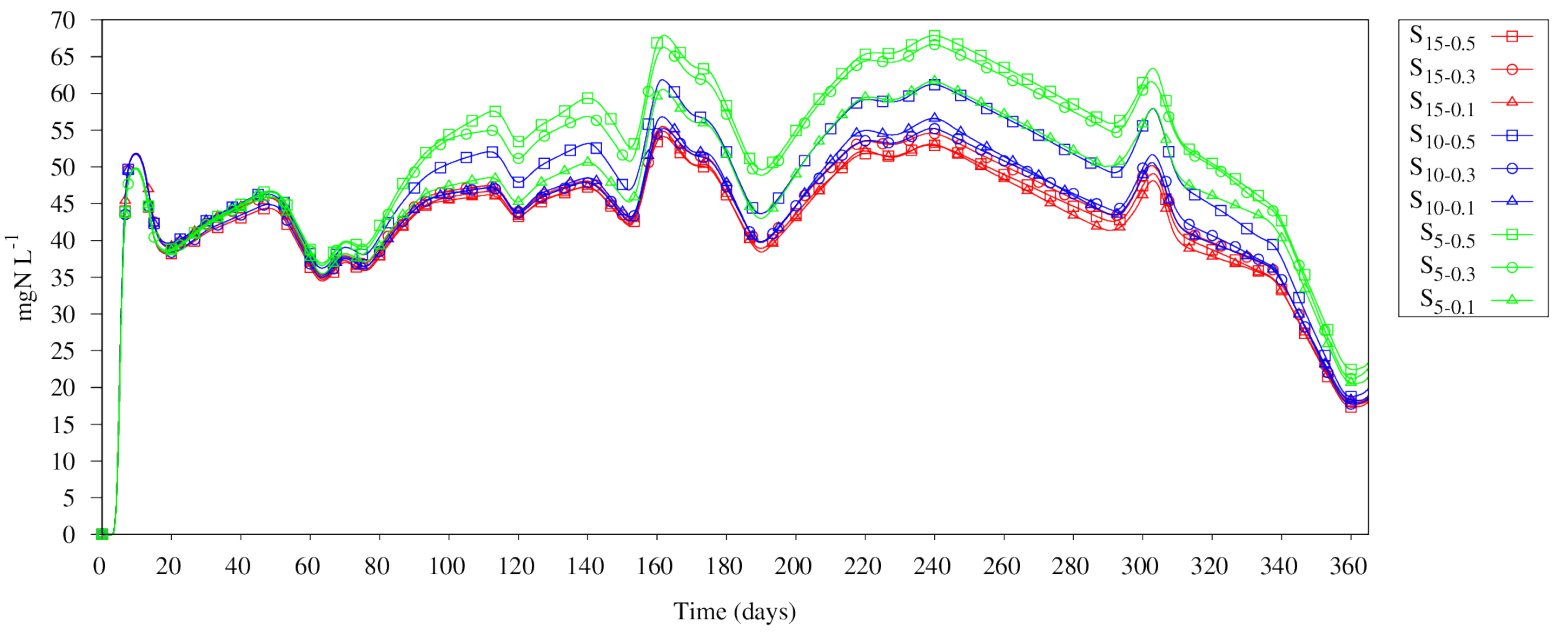

Figure 8: Effluent $S_{N H}$ concentrations obtained with the combinations of $M_{b i o \_m a x}$ and $M_{\text {cap }}$ shown in Table 7. 